\title{
Evaluation of MiR-34 Family and DNA Methyltransferases 1, 3A, 3B Gene Expression Levels in Hepatocellular Carcinoma Following Treatment with Dendrosomal Nanocurcumin
}

\author{
Fatemeh Chamani, Majid Sadeghizadeh*, Mahbobeh Masoumi, Sadegh \\ Babashah
}

\begin{abstract}
Hepatocellular carcinoma $(\mathrm{HCC})$ is the most common primary malignancy of the liver making up more than 80 percent of cases. It is known to be the sixth most prevalent cancer and the third most frequent cause of cancer related death worldwide. Epigenetic regulation constitutes an important mechanism by which dietary components can selectively activate or inactivate target gene expression. The miR-34 family members including mir-34a, mir-34b and mir-34c are tumor suppressor micro RNAs, which are expressed in the majority of normal tissues. Several studies have indicated silencing of miR-34 expression via DNA methylation in multiple types of cancers. Bioactive nutrients like curcumin (Cur) have excellent anticarcinogenic activity and minimal toxic manifestations in biological systems. This compound has recently been determined to induce epigenetic changes. However, Cur is lipophilic and has a poor systemic bioavailability and poor absorption. Its bioavailability is increased through employing dendrosome nanoparticles. The aim of the current study was to investigate the effect of dendrosomal nanocurcumin (DNC) on expression of mir-34 family members in two HCC cell lines, HepG2 and Huh7. We performed the MTT assay to evaluate DNC and dendrosome effects on cell viability. The ability of DNC to alter expression of the mir-34 family and DNA methyltransferases (DNMT1, DNMT3A and 3B) was evaluated using semi-quantitative and quantitative PCR. We observed the entrance of DNC into HepG2 and Huh7 cells. Gene expression assays indicated that DNC treatment upregulated mir34a, mir34b and mir34c expression $(\mathrm{P}<0.05)$ as well as downregulated DNMT1, DNMT3A and DNMT3B expression $(\mathrm{P}<0.05)$ in both HepG2 and Huh7 cell lines. DNC also reduced viability of Huh7 and HepG2 cells through restoration of miR-34s expression. We showed that DNC could awaken the epigenetically silenced miR-34 family by downregulation of DNMTs. Our findings suggest that DNC has potential in epigenetic therapy of HCC.
\end{abstract}

Keywords: Hepatocellular carcinoma - MTT assay - dendrosomal nanocurcumin - microRNA-34 - DNA methyltransfrase

Asian Pac J Cancer Prev, 17, Cancer Control in Western Asia Special Issue, 219-224

\section{Introduction}

Hepatocellular carcinoma (HCC) is the most common primary malignant cancer of the liver with high rates of lethality in the world (Lope et al; 2012). It is the fifth most common cancer worldwide and the third leading cause of death from cancer in the world, with resistance to chemotherapy and poor prognosis (Yao et al;. 2009). It is as an epithelial tumor that is originated from stem cells or mature hepatocytes (Malenstein et al; 2011). The major risk factors of HCC are viral hepatitis infection, chronic alcohol consumption, fungal toxins (aflatoxins), nonalcoholic fatty liver disease (NAFLD), hemochromatosis and steroid hormone(Yang et al., 2010, Gavilán et al., 2013). HCC occurs commonly and with increasing frequency in developing countries
(85\%) such as sub-Saharan Africa and Southeast Asia that viral hepatitis infection is epidemic (El-Serag 2011). Despite advances of treatment in recent years, HCC has a high resistance against conventional therapy. Therefore, new diagnostic and therapeutic approaches are urgently required. Discovery of genetic and epigenetic events in HCC development including genetic variations, histone modifications, DNA methylation and microRNAs have attracted great interest in this matter (Anestopoulos et al; 2015)

MicroRNAs (miRNAs) are a class of small non-coding RNAs with approximately 22-24 nucleotides in length and negatively regulate post translation of genes involved in cellular processes such as cell proliferation, apoptosis, invasion, metastasis, angiogenesis, differentiation, developmental timing and organ development (Lee et al; 
2009, Law et al; 2011, Li et al; 2014). The miR-34 family members including mir34a, mir-34b and mir-34c are tumor suppressor micro RNAs that express in the majority of normal tissues. In the human genome, the miR-34a is encoded on chromosome 1, and the homologs miR-34b and miR-34c are encoded on chromosome 11( $\mathrm{Li}$ et al; 2014). The promoter region of miR-34 family consists of CPG islands. Methylation of these islands reduces the expression levels of miR-34 family in a large number of tumors including breast, colorectal, melanoma and HCC (Gopalan et al., 2014, Li et al., 2014). It is indicated that the demethylating agents are able to activate the expression of these genes and inhibit the growth of tumor in vitro (Nalls et al., 2011, Kong et al., 2012, Roy et al., 2012). DNA methylation is catalyzed by the DNA methyltransferase enzymes (DNMTs) by using S-adenosyl-L-methionine (SAM) as a methyl donor group. Different isoforms of this enzyme including DNMT1, DNMT2, DNMT3a, DNMT3b and DNMT3L exist in mammals (Klose et al., 2006, You et al., 2012).

DNA methylation as an alternative mechanism leads to a decrease in expression of miR-34 family in a variety of cancers such as colorectal cancer, ovarian cancers, oral cancer and malignant pleural mesothelioma (Kozaki et al., 2008, Corney et al., 2010, Kalimutho et al., 2011, Kuboet al., 2011). Several evidences have shown that populations with greater reliance on spices, vegetables and fruits in the diet experience lower risk for cancers. Many of the herbal sources, currently used to treat cancer or other diseases, are naturally multi targeting, safer, inexpensive and immediately available (Gupta et al; 2011). Curcumin is a biphenyl compound derived from rhizome of Curcuma longa and commonly called turmeric. It has excellent antioxidant, anti-inflammatory and anti-tumorigenic properties (Altunbas et al; 2011). However, the significant therapeutic properties of cur have been hindered by its low bioavailability.

Numerous approaches including the use of liposomes, nanoparticles, micelles, adjuvants and phospholipid complexes have been explored to increase the bioavailability of Cur (Anand et 1; 2007). Here, we employed dendrosome nanoparticles to increase the bioavailability and solubility of this agent in comparison with other carriers such as micelles, liposomes and phospholipids. Dendrosome nanoparticles, previously synthesized by our group (Sadeghizadeh et al; 2008) have several advantages such as stability, electrically neutral, biodegradability, spherical structure, non-toxic and easy preparation. Previous studies by our group have shown that dendrosomal nano curcumin (DNC) increase uptake of curcumin into cancer cells without any toxic effects on normal cells (Babaei et al., 2012, Tahmasebi Mirgani et al., 2014). Therefore, the aim of the current study is to test the gene expression levels of miR-34 family members and DNMTs in Huh7 and HepG2 cells following treatment with DNC.

\section{Materials and Methods}

\section{DNC preparation}

To provide the DNC, curcumin powder (Purchased from Sigma-Aldrich Company,USA) and nano-carrier (a gift from Institute of Biochemistry and Biophysics, University of Tehran, Iran ) based on optimized protocols as described in the previous findings (Babaei et al., 2012, Tahmasebi Mirgani et al., 2014), were combined together. To provide the appropriate concentration of DNC, it is diluted with Phosphate buffered saline (PBS) and stored in refrigerator.

\section{Cell lines and culture condition}

In this study, two cancer cell lines including Huh7 and HepG2 cells (from Pasteur Institute, Tehran, Iran) were cultured in Dulbecco's modified Eagle's medium (DMEM; GIBCO ${ }^{2}$, USA) with $10 \%$ fetal bovine serum (FBS; GIBCO ${ }^{\circ}$, USA), $100 \mathrm{U} / \mathrm{ml}$ penicillin and 100 $\mathrm{mg} / \mathrm{ml}$ streptomycin (LifeTechnologies). All cells were incubated at $37^{\circ} \mathrm{C}$ and $5 \%$ carbon dioxide.

\section{Cell viability test}

Cell viability is used for drug screening and cytotoxicity tests of chemicals. This was measured by 3-(4,5-dimethylthiazol-2-yl)-2,5-diphenyltetrazolium bromide (MTT) assay according to the manufacturer's instructions (Sigma-Aldrich,USA). Briefly, identical numbers of hepatoma cells were seeded in 96-well plates triplicate in a final volume of $200 \mu$ l medium with $10 \%$ FBS and incubated overnight at $37^{\circ} \mathrm{C}$ and $5 \% \mathrm{CO} 2$. Huh7 and HepG2 cell lines were treated with varying concentrations of DNC for $24 \mathrm{~h}$ and $48 \mathrm{~h}$. A complete culture medium without cells was used as a blank. After removing the medium of each well, $20 \mu$ l of MTT $(5 \mathrm{mg} /$ $\mathrm{ml}$ ) was added to each well and plates were incubated

Table 1. Primers Sequence Used for q-PCR

\begin{tabular}{|c|c|c|c|c|}
\hline Gene & Chromosoe Location & Primer & Sequence & Ampliqon length \\
\hline \multirow[t]{2}{*}{ DNMT1 } & $19 \mathrm{p} 13.2$ & Forward & 5'-GAAGGAGCCCGTGGATG-3' & $233 \mathrm{bp}$ \\
\hline & & Reverse & 5'-GTTGATGTCTGCGTGGTAG-3' & \\
\hline \multirow[t]{2}{*}{ DNMT3A } & $2 \mathrm{p} 23$ & Forward & 5'-TACGCACCACCTCCAC-3' & $110 \mathrm{bp}$ \\
\hline & & Reverse & 5'-AGATGTCCTCAATGTTCC-3' & \\
\hline \multirow[t]{2}{*}{ DNMT3B } & $20 \mathrm{q} 11.2$ & Forward & 5'-CGACCTCACAGACGACAC-3' & $170 \mathrm{bp}$ \\
\hline & & Reverse & 5'-TTCCAAACTCCTTCCCATCC-3' & \\
\hline \multirow[t]{2}{*}{ GAPDH } & $12 \mathrm{p} 13$ & Forward & 5'GTGAACCATGAGAAGTATGACAAC3' & $123 \mathrm{bp}$ \\
\hline & & Reverse & 5'-CATGAGTCCTTCCACGATACC-3 & \\
\hline
\end{tabular}

bp: base pair 
(a)

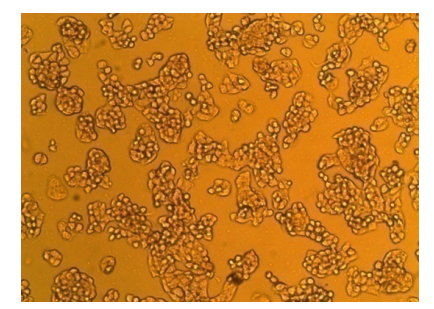

(b)

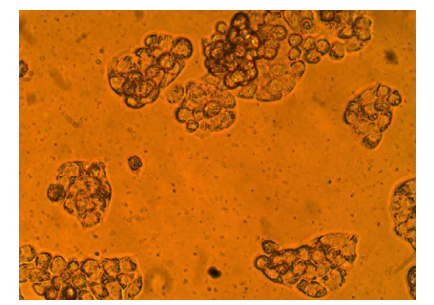

(c)

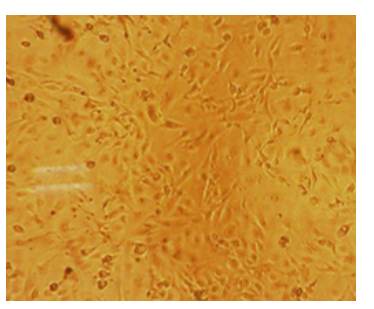

(d)

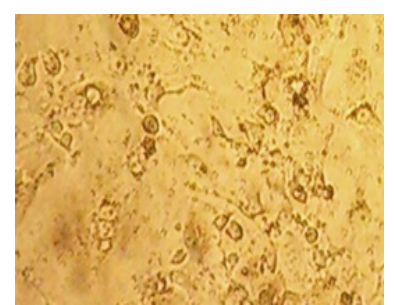

Figure 1. Microscopic Visualization of DNC. HepG2 and HuH7 Cells were Treated with DNC: (a) HepG2 Control,(b) HepG2 Treated for 48h,(c) HuH7 Control,(d) HuH7 Treated for48h. HCC Cells Lines Treated with DNC Showed a Time and Dose Dependent Reduced Viability Compared with Untreated Cell (200× Magnification).

for another $4 \mathrm{~h}$. Then the supernatant was removed and the insoluble formazan crystals were dissolved in $200 \mu \mathrm{l}$ of dimethyl sulfoxide (DMSO). The absorbance intensity was measured at $570 \mathrm{~nm}$ by a $96-$-well plate reader (TECAN, Switzerland). The results were expressed as percentage of cell viability against the untreated control cells. Afterwards, 50\% inhibitory concentrations (IC50) that means concentration where half of the cells were alive, were calculated by standard curve method.

\section{$R N A$ extraction and reverse transcription}

According to the amount of IC50 obtained from MTT assay, Huh7 and HepG2 cell lines were treated with DNC. After $24 \mathrm{~h}$ and $48 \mathrm{~h}$ incubation, total RNA was extracted from each group with TRIzol ${ }^{\circ}$ reagent (Life Technologies). RNA quantity was analyzed using NanoDrop 1,000 (Thermo, USA). To verify the RNA quality, the existence of $18 \mathrm{~s}, 28 \mathrm{~s}$ and $5 \mathrm{~s}$ were analyzed on agarose gel. Complementary DNA (cDNA) was synthesized by PrimeScriptTMRT reagent kit (Takara Bio Inc,Shiga, Japan). Primers sequence for house keeping gene glyceraldehyde3-phosphatedehydrogenase (GAPDH) and DNMT1, DNMT3A and DNMT3B genes are indicated in Table1.

Table2: IC50 of DNC for two hepatoma cell lines

\begin{tabular}{lcr}
\hline Cell lines & IC50 for $24 \mathrm{~h}$ & IC50 for $48 \mathrm{~h}$ \\
\hline Huh7 & 25 & 15 \\
HepG2 & 30 & 26 \\
\hline
\end{tabular}

Semi quantitative reverse transcription PCR (sqRT-PCR)

After reverse transcription reaction in order to ensure the accuracy and specificity of the primers, reverse transcription PCR reaction was performed on the RT product. PCR was done using Maste Mix (Ampliqon, Denmark) at the following conditions: denaturation at 94 ${ }^{\circ} \mathrm{C}$ for 30 seconds in a cycle and annealing at $60^{\circ} \mathrm{C}$ for 30 seconds in 35 cycles. PCR products were separated on $2 \%$ agarose gel electrophoresis and were observed by using ultraviolet rays after staining with ethidium bromide.

Real-Time quantitative reverse transcription-PCR (Real-Time qRT-PCR)

Real-time PCR was performed using the SYBR ${ }^{\circledR}$ Premix Ex Taq TM II (Takara Bio Inc.,Shiga,Japan). The relative amount of DNMTs gene expression levels were normalized to the expression of GAPDH as house-keeping gene. Relative gene expression was calculated using the comparative cycle threshold $(\mathrm{RQ}=2-\Delta \Delta \mathrm{CT})$ method.

\section{MiRNA expression analysis}

RNA was extracted from either control or treated group using TRIzol ${ }^{\circledR}$ reagent (Life Technologies). Due to the small size of the microRNA, a poly A nucleotide tail was added to the miRNAs using polyA polymerase. In the reverse transcription step, cDNA is reverse transcribed using specific reverse primers and reverse transcriptase enzyme using MiR-Amp kit (Pars Genome,Tehran,Iran), according to the manufacturer's instruction. In the PCR step, PCR products are amplified from cDNA samples using $5 \times$ HOT FIRE POL ${ }^{\circledR}$ EvaGreen ${ }^{\circledR}$ qPCR Mixplus ROX (Solis Bio Dyne,Tartu,Estonia) kit. The expressions
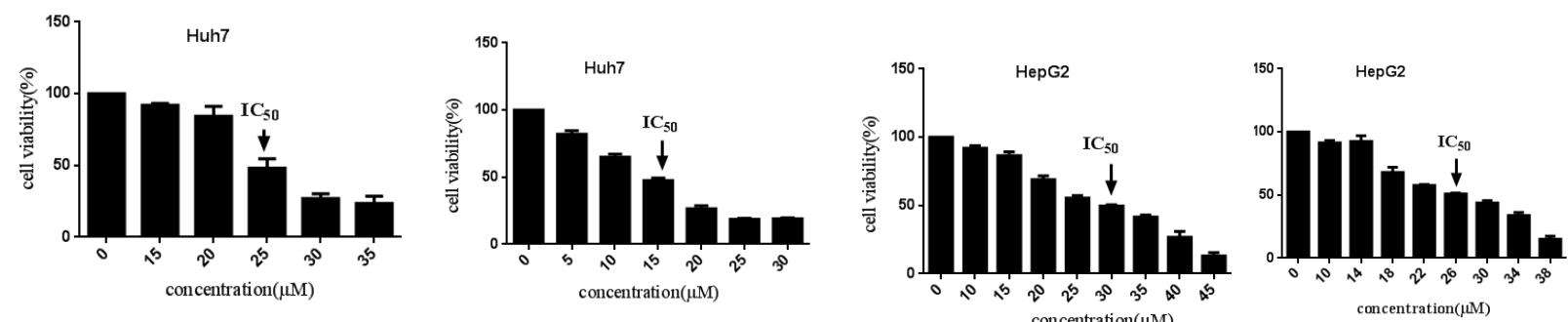

Figure 2. Cytotoxic Effects of DNC on Cancerous Cells. HepG2 and HuH7 Cells were Treated with Different Concentrations of DNC:(a) HuH-7 (0-35 $\mu \mathrm{M})$ for 24h,(b) HuH7 (0-30 $\mu \mathrm{M})$ for 48h(c) HepG2 (0-45 $\mu \mathrm{M})$ for 24h and (d) HepG2 $(0-38 \mu \mathrm{M})$ for $48 \mathrm{~h}$ were Checked for Their Proliferation by MTT Assay. DNC: Dendrosomal Nanocurcumin; IC50, $50 \%$ Inhibitory Concentrations 
a)

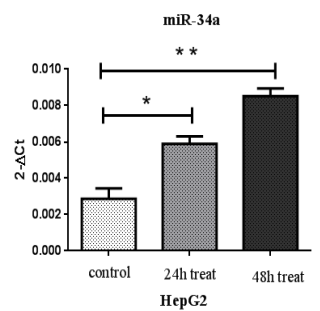

d)

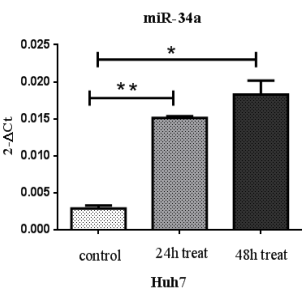

b)

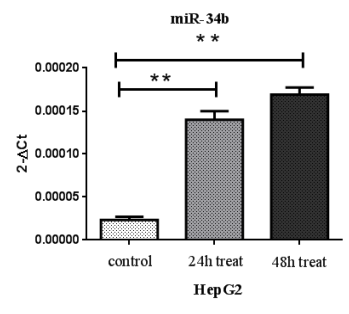

e)

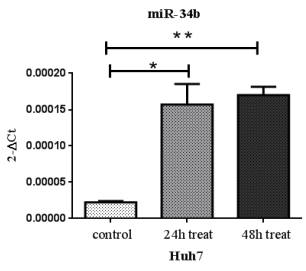

c)

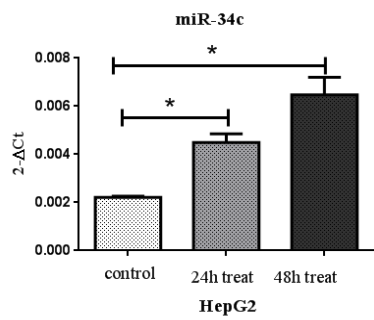

f)

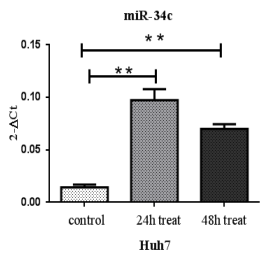

Figure 3. DNC up-Regulates miR-34a, miR-34b and miR-34c in Hepatoma Cell Lines a) miR-34a b) miR-34b and c) miR-34c in HepG2, d) miR-34a e) miR-34b and f) miR-34c in Huh7. Bars Represent Mean and Standard Error of Three Independent Experiments. Control: Non-Treated Sample, ns: Non Significant, ${ }^{*} \mathrm{P}<0.05$, ${ }^{* *} \mathrm{P}<0.01$.

of miR-34a, miR-34b, miR-34c and U6 (reference gene) were analyzed using universal and specific primer sets MiR-Amp kit (ParsGenome, Tehran,Iran). Relative gene expression was calculated using the comparative cycle threshold $(\mathrm{RQ}=2-\Delta \Delta \mathrm{CT})$ method.

\section{Statistical analysis}

All statistical analyses were performed using Prism $\AA 5$ software (Graph Pad Soft-ware, Inc., LaJolla, CA, USA) and Microsoft Excel. Data were presented in one way analysis of variance followed by Newman-Keuls multiple comparison test or Student's t-test to compare expression between two group. The $\mathrm{P}<0.05$ was considered statistically significant.

\section{Results}

Curcumin inhibit hepatoma cancer cell proliferation

The cytotoxic effect of DNC on HepG2 and HuH-7 cell lines could be seen microscopically as vesicular bodies in
DNC treated cells in Figure $1 \mathrm{~b}$ and d compared to control ones in Fig. 1 a and c. We also performed a MTT assay to study the effects of curcumin on the proliferation of Huh7 and HepG2 hepatoma cell lines. These cell lines were treated with multiple concentrations of curcumin for either $24 \mathrm{~h}$ or $48 \mathrm{~h}$. As shown in Figure $2 \mathrm{a}$ and b, $25 \mu \mathrm{M}$ and $15 \mu \mathrm{M}$ concentrations of DNC killed $50 \%$ of Huh7 cells after $24 \mathrm{~h}$ and $48 \mathrm{~h}$, respectively. So IC50 values for DNC in Huh7 cells is $25 \mu \mathrm{M}$ for $24 \mathrm{~h}$ and $15 \mu \mathrm{M}$ for treated 48 h. Furthermore, as shown in Fig. $2 \mathrm{c}$ and d, $30 \mu \mathrm{M}$ and $26 \mu \mathrm{M}$ concentrations of DNC killed $50 \%$ of HepG2 cells after $24 \mathrm{~h}$ and $48 \mathrm{~h}$, respectively. So the IC50 value of DNC for HepG2 cell line with in $24 \mathrm{~h}$ was about $30 \mu \mathrm{M}$ which relatively reduced to $26 \mu \mathrm{M}$ in $48 \mathrm{~h}$ (Table2). Administration of DNC leaded to significant cell death in Huh7 and HepG2 cell lines, in a time and dose dependent manner.

DNC up-regulates $m i R-34 a, m i R-34 b$ and $m i R-34 c$ in hepatoma cell lines a)

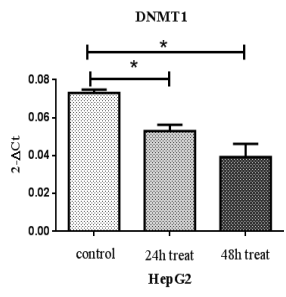

d)

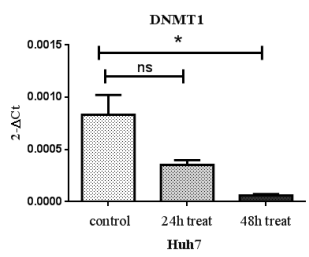

b)

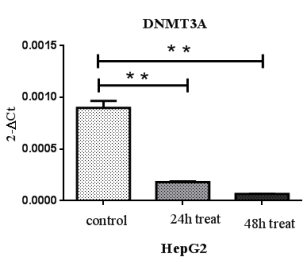

e)

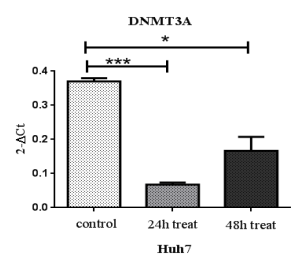

c)

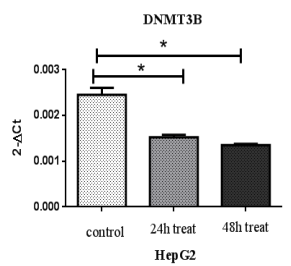

f)

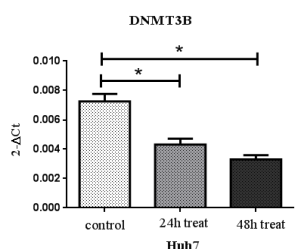

Figure 4. DNC Down-Regulates DNMT1, DNMT3A and DNMT3B in Hepatoma Cell Lines .a) DNMT1 b) DNMT3A and c) DNMT3B in HepG2, d) DNMT1 e)DNMT3A and f) DNMT3B in Huh7. Bars Represent Mean and Standard Error of Three Independent Experiments. Control: Non-Treated Sample, ns: Non Significant, ${ }^{*} \mathrm{P}<0.05,{ }^{* *} \mathrm{P}<0.01,{ }^{* * *}$ 
To investigate the effect of DNC on miR-34 family expression, Huh7 and HepG2 cell lines were treated with DNC after $24 \mathrm{~h}$ and $48 \mathrm{~h}$ and alterations in the genes expression of miR-34 family were evaluated by Real-Time qRT-PCR as shown in Fig3. Our current data demonstrated that DNC significantly induced the expression of miR-34a, miR-34b and miR-34c relative quantification to reference gene U6 both Huh7 and HepG2 $(\mathrm{P}<0.05)$.

\section{Dendrosomal nanocurcumin down-regulates DNMTs in hepatoma cell lines}

Huh7 and HepG2 cell lines were treated with DNC for $24 \mathrm{~h}$ and $48 \mathrm{~h}$ then the alterations in the genes expression of DNMTs and GAPDH were evaluated by Real-Time qRT-PCR (Fig4). Our current data demonstrated that DNC significantly,reduced the expression of DNMT1, DNMT3A and DNMT3B relative quantification to reference gene GAPDH both Huh7 and HepG2 $(\mathrm{P}<0.05)$.

\section{Discussion}

Bioactive nutrients including curcumin (a biphenyl compound derived from rhizome) offer great potential in causing cancerous cell death. In current study, using MTT assay, we indicated that HCC cells lines treated with DNC showed a time and dose dependent reduced viability compared with untreated cells. We used DNC with higher bioavalibility compared to curcumin. The dendrosomes were shown to be safe for entrance of curcumin to cancer cells and only DNC leads to death of cancer cells and dendrosome has facilitated delivery of curcumin to cancer cells (Babaei et al; 2012, Tahmasebi Mirgani et al; 2014, Zamani et al; 2015).

The miR-34 family members including mir34a, mir34b and mir34c acts as tumor suppressor microRNAs. Hence, their reduction or loss of expression was reported in a large number of tumors including breast, colorectal, melanoma and HCC (Gopalan et al; 2014, Li et al; 2014). However, no studies have been performed to determine whether miR-34 family could be re-expressed in hepatoma cancer by DNC. We found that DNC upregulated mir34a, mir34b and mir34c expression $(\mathrm{P}<0.05)$ in both HepG2 and Huh7 cell lines. We indicated that these upreulation is the result of DNMTs down-regulation.

Our previous work showed that DNC could induce the apoptosis of cancer cells by down-regulation of DNMTs expression following the overexpress of tumor suppressor genes (Babaei et al; 2012, Tahmasebi Mirgani et al; 2014, Zamani et al; 2015).

In conclusion, we provide evidence that administration of DNC inhibits proliferation of HCC cells through increasing the expression of miR-34a, miR-34b and $\mathrm{miR}-34 \mathrm{c}$.

Furthermore, our findings demonstrate that the expression of DNMT1, DNMT3A and DNMT3B is greatly down-regulated under treated with $\mathrm{DNC}$, suggesting that DNC restores the expression of mir-34 family members via down-regulation of these enzymes. These findings suggest that DNC potentially could be used in clinic especially for HCC therapy.

\section{Conflict of interest}

None of the authors have any conflicts of interest in this work.

\section{Acknowledgements}

The authors gratefully acknowledge the research and Development Center for Biotechnology of Tarbiat Modares University, Tehran, Iran, the Faculty of Biological Sciences, Tarbiat Modares University, Tehran, Iran, Deputy of Research, Ministry of Health and Medical Education, Tehran, Iran and for supporting us in performing the project and MS M.Motavaf ,PhD student of Molecular Genetics, Department of Genetics, Faculty of Biological Sciences, Tarbiat Modares University, Tehran, Iran for editing this article.

\section{References}

Altunbas A, Lee SJ, Rajasekaran SA, Schneider JP, Pochan DJ (2011). Encapsulation of curcumin in self-assembling peptide hydrogels as injectable drug delivery vehicles. Biomaterials, 32, 5906-14.

Anand P, Kunnumakkara AB, Newman RA, Aggarwal BB (2007). Bioavailabilityofcurcumin:problemsandpromises. Mol Pharm, 4, 807-18

Anestopoulos I, Voulgaridou GP, Georgakilas AG, et al (2015). Epigenetic therapy as a novel approach in hepatocellular carcinoma. J Pharmacol Exp Ther, 145, 103-19.

Babaei E, Sadeghizadeh M, Hassan ZM, et al (2012). Dendrosomal curcumin significantly suppresses cancer cell proliferation in vitro and in vivo. Immuno pharmacol, 12, 223-6.

Corney DC, Hwang CI, Matoso A, et al (2010). Frequent downregulation of miR-34 family in human ovarian cancers. Clin Cancer Res, 16, 1119-28.

De Lope CR, Tremosini S, Forner, Reig AM, Bruix J (2012). Management of HCC. J HepatoL, 8, 75-87.

E-Serag HB, Rudolph KL (2011). Hepatocellular carcinoma. $N$ Engl J Med, 365, 1118-27

Gavilán JCOG, Arnedo R, Puerta S (2013). Predictive factors of risk of hepatocellular carcinoma in chronic hepatitis $\mathrm{C}$. Eur J Intern Med, 24, 846-51.

Gopalan V, Pillai S, Ebrahimi F, et al (2014). Regulation of microRNA-1288 in colorectal cancer: altered expression and its clinicopathological significance. Mol Carcinog, 53, 36-44.

Gupta SC, Kim JH, Kanappan R, et al (2011). Role of nuclear factor-kb-mediated inflammatory pathways in cancer-related symptoms and their regulation by nutritional agents. Exp Biol, 236, 658-71.

Kalimutho M, Di Cecilia S, Del Vecchio Blanco G, et al (2011). Epigenetically silenced $\mathrm{miR}-34 \mathrm{~b} / \mathrm{c}$ as a novel faecalbased screening marker for colorectal cancer. $r J$ Cancer, 104, $1770-8$

Klose RJ, Bird AP (2006). Genomic DNA methylation: the mark and its mediators. Trends Biochem Sci, 31, 89-97.

Kong D, Heath E, Chen W, et al (2012). Epigenetic silencing of miR-34a in human prostate cancer cells and tumor tissue specimens can be reversed by BR-DIM treatment. Am $J$ Transl Res, 4, 14-23.

Kozaki K, Imoto I, Mogi S, Omura K, Inazawa J (2008). Exploration of tumorsuppressivemicroRNAs silenced by DNA hypermethylation in oral cancer. Cancer Res, $6 \mathbf{8}$, 2094-105. 
Kuboet T, Toyooka S, Tsukuda K, et al.. (2011). Epigenetic silencing of microRNA-34b/c plays an important role in the pathogenesis of malignant pleural mesothelioma. Clin Cancer Res, 17, 4965-74.

Law PT, Wong N (2011). Emerging roles of microRNA in the intracellular signaling networks of hepatocellular carcinoma. $J$ Gastroentero Hepatol, 26, 437-49.

Lee YS, Dutta A (2009). MicroRNAs in cancer. Annu Rev Pathol, 4, 199-227.

Li XJ, Ren ZJ, Tang JH (2014). MicroRNA-34a: a potential therapeutic target in human cancer. Cell Death Dis, 5, e1327.

Nalls D, Tang SN, Rodova M, Srivastava RK, Shankar S (2011). Targeting epigenetic regulation of miR-34a for treatment of pancreatic cancer by inhibition of pancreatic cancer stem cells. PLoS One, 6, e24099.

Roy S, Levi E, Majumdar A, Sarkar FH (2012). Expression of miR-34 is lost in colon cancer which can be re-expressed by a novel agent CDF. J Hematol Oncol, 5, 1756-62.

Sadeghizadeh M, Ranjbar B, Damaghi M, et al (2008). Dendrosomes as novel gene porters III. $J$ Chem Technol Biotechnol, 6, 912-20.

Tahmasebi Mirgani M, Isacchi B, Sadeghizadeh M, et al (2014). Dendrosomal curcumin nano formulation downregulates pluripotency genes via miR-145 activation in U87 MG glioblastoma cells. Int J Nanomed, 9, 403-17.

Van Malenstein H, Van Pelt J, Verslype C (2011). Molecular classification of hepatocellular carcinoma anno 2011. Eur $J$ Cancer, 47, 1789-97

Yang H, Sherman IM, Su J, et al (2010). Nomograms for risk of hepatocellular carcinoma in patients with chronic hepatitis B virus infection. J Clin Oncol, 28, 2437-44.

Yao z, Mishra L (2009). Cancer stem cells and hepatocellular carcinoma. Cancer Biol, 8, 1691-8.

You JS, Jones PA (2012). Cancer genetics and epigenetics: two sides of the same coin?. Cancer Cell, 22, 9-20.

Zamani M, Sadeghizadeha M, Behmanesh M, Najafi F(2015). Dendrosomal curcumin increases expression of the long non-coding RNA gene MEG3 via up-regulation of epi-miRs in hepatocellular cancer. Phytomedicine, 22, 961-7. 